\title{
Stability analysis of snake robot locomotion based on Poincaré maps
}

\author{
Pål Liljebäck, Kristin Y. Pettersen, Øyvind Stavdahl, and Jan Tommy Gravdahl
}

\begin{abstract}
This paper presents an analysis of snake locomotion that explains how non-uniform viscous ground friction conditions enable snake robots to locomote forward on a planar surface. The explanation is based on a simple mapping from link velocities normal to the direction of motion into propulsive forces in the direction of motion. From this analysis, a controller for a snake robot is proposed. A Poincaré map is employed to prove that all state variables of the snake robot, except for the position in the forward direction, trace out an exponentially stable periodic orbit.
\end{abstract}

\section{INTRODUCTION}

Inspired by biological snakes, snake robots carry the potential of meeting the growing need for robotic mobility in challenging environments. Snake robots consist of serially connected modules capable of bending in one or more planes. The many degrees of freedom of snake robots make them difficult to control, but provides traversability in irregular environments that surpasses the mobility of the more conventional wheeled, tracked and legged forms of robotic mobility.

There are several reported works aimed at analysing and understanding snake locomotion. Gray [1] conducted empirical and analytical studies of snake locomotion already in the 1940s. Hirose [2] studied biological snakes and developed mathematical relationships characterizing their motion, such as the serpenoid curve. Other works on modelling and control of snake robots include [3]-[10]. All these works provide interesting analyses and simulation results that contribute to the understanding of snake locomotion. However, in the authors' opinion, previously published research on snake robots has not presented an explicit mathematical description that easily explains how a snake robot possessing non-uniform friction properties is able to produce forward motion on a flat surface (such a description represents the first contribution of this paper).

The method of Poincaré maps [11] represents a widely used tool for proving the existence and stability of periodic orbits of dynamical systems [12]. To the authors' best knowledge, Poincaré maps have never before been employed for the analysis of snake locomotion (such an analysis represents the second contribution of this paper).

This paper provides two contributions. The first contribution is the development of a simple relationship between link velocities normal to the direction of motion and propulsive forces in the direction of motion that explains how snake robots influenced by non-uniform viscous ground friction is able to locomote forward on a planar surface. The second

Affiliation of Pål Liljebäck is shared between the Department of Engineering Cybernetics at the Norwegian University of Science and Technology, NO-7491 Trondheim, Norway, and SINTEF ICT, Dept. of Applied Cybernetics, N-7465 Trondheim, Norway. E-mail: Pal. Liljebackasintef.no

K. Y. Pettersen, Øyvind Stavdahl, and Jan Tommy Gravdahl are with the Department of Engineering Cybernetics at the Norwegian University of Science and Technology, NO-7491 Trondheim, Norway. E-mail: $\quad$ Kristin.Y.Pettersen, Oyvind.Stavdahl, Tommy. Gravdahl\} ditk.ntnu.no

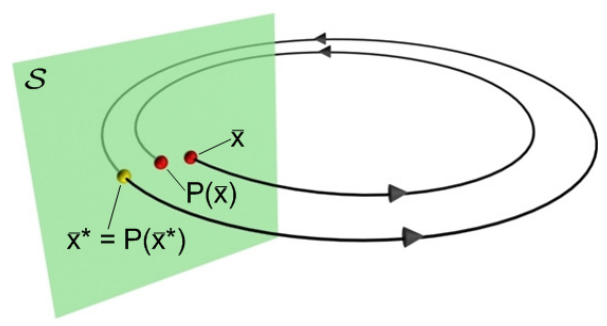

Fig. 1. Illustration of the Poincaré map corresponding to a Poincaré section $\mathcal{S}$.

contribution is the use of a Poincaré map to study stability properties of snake robot locomotion. More specifically, a Poincaré map is employed to prove that all state variables of a snake robot, except for the position in the forward direction, trace out an exponentially stable periodic orbit when the proposed controller is applied.

The paper is organized as follows. Section II gives an introduction to Poincaré maps. Section III presents a mathematical model of a planar snake robot. Section IV presents an analysis of snake locomotion. Section V proposes a controller for the snake robot. Stability analysis of the snake locomotion based on a Poincaré map is presented in Section VI. Finally, Section VII presents concluding remarks.

\section{INTRODUCTION TO POINCARÉ MAPS}

This section gives an informal presentation of the Poincaré map since this is used as a stability analysis tool in Section VI. The presentation is based on [11] and [12].

\section{A. General description of Poincaré maps}

The Poincaré map represents a widely used tool for analysing the existence and stability of periodic orbits of dynamical systems. Consider an autonomous (not explicitly dependent on time) $n$-dimensional dynamical system of the form

$$
\dot{x}=f(x) \quad, \quad x \in \mathbb{R}^{n}
$$

where $f(x)$ is assumed to be continuously differentiable. Assume that the solution of this differential equation for a particular initial condition is a limit cycle. This means that the flow of $x$ in the $n$-dimensional state space will return to the initial condition after a time $T$, corresponding to the period of the limit cycle.

We now define an $(n-1)$-dimensional hyperplane $\mathcal{S}$ (called a Poincaré section) such that the limit cycle intersects and passes through $\mathcal{S}$ at some instant in time. We denote by $\bar{x} \in \mathbb{R}^{n-1}$ the $(n-1)$-dimensional state vector when $x$ is constrained to $\mathcal{S}$. The point on $\mathcal{S}$ where the limit cycle intersects $\mathcal{S}$ is denoted $\bar{x}^{*} \in \mathbb{R}^{n-1}$. Assume now that we initialize (1) on the hyperplane $\mathcal{S}$ somewhere close to $\bar{x}^{*}$. Due to the continuity of the solutions of (1) with respect to the initial condition, the flow of $x$ will, in approximately 
$T$ seconds, return to and intersect $S$ somewhere close to $\bar{x}^{*}$. This is illustrated in Fig. 1. The mapping from an initial point $\bar{x}$ on $\mathcal{S}$ to the next point where the flow of $x$ intersects $\mathcal{S}$ is called the Poincaré map and is denoted by $P(\bar{x}) \in \mathbb{R}^{n-1}$. The Poincaré map is in other words a function that accepts an initial point on a Poincaré section as input and outputs where the Poincaré section will be intersected next by the flow of $x$. This is written more formally as $P: \mathcal{S} \rightarrow \mathcal{S}$. The point $\bar{x}^{*}$ is called a fixed point of the Poincaré map since the Poincaré map maps $\bar{x}^{*}$ back to itself. This is also illustrated in Fig. 1. We only consider one-sided Poincaré maps, i.e. we only consider crossings of $\mathcal{S}$ in directions corresponding to the direction of $\dot{x}$ when $x$ initially left $\mathcal{S}$.

The Poincaré map can be interpreted as a discrete-time system with an $(n-1)$-dimensional state space that evolves on the Poincare section. This is seen by denoting by $\bar{x}[k] \in$ $\mathcal{S}$ the point of the $k$-th intersection with $\mathcal{S}$ by the flow of $x$. The Poincaré map may then be written as

$$
\bar{x}[k+1]=P(\bar{x}[k]) \quad, \bar{x}[0] \in \mathcal{S}
$$

which is a discrete-time system.

The usefulness of the Poincaré map for stability analysis lies in the fact that local exponential stability of the fixed point $\bar{x}^{*}$ on the Poincare section is equivalent to local exponential stability of the underlying periodic orbit [12], i.e. nearby orbits converge exponentially to the periodic orbit. Note that the stability is only asymptotic (i.e. not exponential) if $f(x)$ in (1) is continuous, but not continuously differentiable [12]. The problem of determining if a periodic orbit of the system (1) is exponentially stable is, in other words, reduced to determining if $\bar{x}^{*}$ is an exponentially stable equilibrium point of the discrete-time system in (2), which is a much simpler problem to solve. A significant drawback of Poincare maps is that they provide little insight into properties of the system dynamics.

Note that the method of Poincaré maps may also be applied to non-autonomous periodic systems, i.e. systems of the form $\dot{x}=f(x, t)$, by incapsulating the time $t$ in an augmented periodic state variable $\beta=2 \pi t / T$. This is performed for the snake robot in Section VI-A.

\section{B. Practical application of Poincaré maps}

This section provides an informal description of the practical use of Poincaré maps. The aim is to show how this method can be employed in practice in order to investigate the stability properties of a time-periodic dynamical system.

1) Calculating the Poincaré map: It is difficult to determine the Poincaré map analytically since it requires the solution of the differential equation (1). However, the Poincaré map of (1) is simply the forward integration of this differential equation. It is therefore possible to compute the Poincaré map $P\left(\bar{x}_{0}\right)$ numerically by initializing (1) on $\mathcal{S}$ at $\bar{x}_{0}$ and simulating (1) until $\mathcal{S}$ is intersected. The state corresponding to this intersection is the Poincare map $P\left(\bar{x}_{0}\right)$.

2) Locating fixed points of the Poincaré map: The easiest way of locating a fixed point $\bar{x}^{*}$ of the Poincaré map is to simply let the simulation of (1) run until it reaches the steady state. This is called the brute-force approach and has three serious disadvantages. First of all, convergence to the fixed point can be exceedingly slow. Secondly, the method can only locate stable fixed points. Thirdly, it may be difficult to tell when the steady state has been reached.

A more sophisticated method is to exploit the fact that locating $\bar{x}^{*}$ is equivalent to locating zeros of the error function

$$
E(\bar{x})=P(\bar{x})-\bar{x} \quad, \quad E(\bar{x}) \in \mathbb{R}^{n-1}
$$

since we have that $\bar{x}^{*}=P\left(\bar{x}^{*}\right)$. The Newton-Raphson algorithm [11] is a general algorithm for locating zeros of a differentiable function, and may therefore be employed for locating $\bar{x}^{*}$. By starting from an inital guess, $\bar{x}^{k}$, of the fixed point, the Newton-Raphson algorithm calculates a more accurate estimate of $\bar{x}^{*}$ through the formula

$$
\bar{x}^{k+1}=\bar{x}^{k}-J_{E}\left(\bar{x}^{k}\right)^{-1} E\left(\bar{x}^{k}\right)
$$

where

$$
J_{E}=\frac{\partial E}{\partial \bar{x}}=\left[\begin{array}{ccc}
\frac{\partial E_{1}}{\partial \bar{x}_{1}} & \cdots & \frac{\partial E_{1}}{\partial \bar{x}_{n-1}} \\
\vdots & \ddots & \vdots \\
\frac{\partial E_{n-1}}{\partial \bar{x}_{1}} & \cdots & \frac{\partial E_{n-1}}{\partial \bar{x}_{n-1}}
\end{array}\right] \in \mathbb{R}^{n-1 \times n-1}
$$

is the Jacobian of the error function $E(\bar{x})$. The Jacobian $J_{E}\left(\bar{x}^{k}\right)$ can be calculated numerically by defining

$$
d \bar{x}_{i}=\left[\begin{array}{lllllll}
0 & \cdots & 0 & \Delta_{i} & 0 & \cdots & 0
\end{array}\right]^{T} \in \mathbb{R}^{n-1}
$$

where the $i$-th element is non-zero and $\Delta_{i}$ is a small perturbation of $\bar{x}_{i}$ along $S$. Column $i$ of $J_{E}\left(\bar{x}^{k}\right)$ may then be approximated numerically as

$$
\frac{\partial E}{\partial \bar{x}_{i}}\left(\bar{x}^{k}\right) \approx \frac{E\left(\bar{x}^{k}+d \bar{x}_{i}\right)-E\left(\bar{x}^{k}\right)}{\Delta_{i}}
$$

This enables a column-wise construction of $J_{E}\left(\bar{x}^{k}\right)$. If the initial condition is within the basin of attraction of a periodic orbit, the Newton-Raphson algorithm will converge rapidly towards the fixed point $\bar{x}^{*}$.

3) Analysing stability of a periodic orbit: As explained in Section II-A, a fixed point $\bar{x}^{*}$ of the Poincaré map corresponds to a periodic orbit of the underlying dynamical system. Once the fixed point has been found using e.g. the Newton-Raphson algorithm, the stability of the periodic orbit may be tested by investigating if the fixed point is a stable equilibrium point of the Poincaré map, i.e. of the discrete-time system (2). This is done by calculating the Jacobian linearization of the Poincare map about the fixed point, i.e. by calculating the Jacobian $J_{P}\left(\bar{x}^{*}\right)=\left.\frac{\partial P}{\partial \bar{x}}\right|_{\bar{x}=\bar{x}^{*}} \in$ $\mathbb{R}^{n-1 \times n-1} . J_{P}\left(\bar{x}^{*}\right)$ is calculated by following the same procedure as for calculating $J_{E}\left(\bar{x}^{k}\right)$ in (5). The Poincaré map linearized about the fixed point is thereby given as $\bar{x}[k+1]=J_{P}\left(\bar{x}^{*}\right) \bar{x}[k]$. This is a linear discrete-time system which is exponentially stable if the magnitude of all the eigenvalues of $J_{P}\left(\bar{x}^{*}\right)$ are strictly less than one. The fixed point $\bar{x}^{*}$ of the Poincaré map, and thereby also the periodic orbit of the underlying dynamical system, is therefore locally exponentially stable if the magnitude of all the eigenvalues of $J_{P}\left(\bar{x}^{*}\right)$ are strictly less than one.

\section{A MODEL OF THE SNAKE ROBOT}

This section gives a brief introduction to a mathematical model of a planar snake robot previously presented in [10]. 


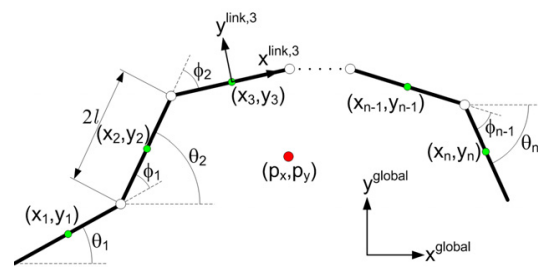

Fig. 2. Kinematic parameters for the snake robot.

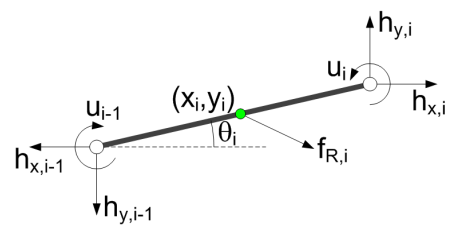

Fig. 3. Forces and torques acting on each link of the snake robot.

\section{A. Notations and defined identities}

The snake robot consists of $n$ links of length $2 l$ interconnected by $n-1$ joints. The mathematical identities defined in order to describe the kinematics and dynamics of the snake robot are described in Table I and illustrated in Fig. 2 and Fig. 3. All $n$ links have the same length, mass, and moment of inertia. The total mass of the snake robot is therefore $n m$. The mass of each link is uniformly distributed so that the link $\mathrm{CM}$ (center of mass) is located at its center point (at length $l$ from the joint at each side).

The following vectors and matrices are used in the subsequent sections:

$$
A:=\left[\begin{array}{lllll}
1 & 1 & & & \\
& \cdot & \cdot & & \\
& & \cdot & \cdot & \\
& & & 1 & 1
\end{array}\right], D:=\left[\begin{array}{ccccc}
1 & -1 & & & \\
& \cdot & \cdot & & \\
& & \cdot & \cdot & \\
& & & 1 & -1
\end{array}\right]
$$

where $A \in \mathbb{R}^{(n-1) \times n}$ and $D \in \mathbb{R}^{(n-1) \times n}$. Furthermore,

$$
e:=\left[\begin{array}{llll}
1 & \cdot & \cdot & 1
\end{array}\right]^{T} \in \mathbb{R}^{n} \quad E=\left[\begin{array}{cc}
e & 0_{n \times 1} \\
0_{n \times 1} & e
\end{array}\right] \in \mathbb{R}^{2 n \times 2}
$$

\begin{tabular}{|l|l|l|}
\hline Symbol & Description & $\begin{array}{l}\text { Associated } \\
\text { vector }\end{array}$ \\
\hline$\theta_{i}$ & Angle between link $i$ and global $x$ axis. & $\theta \in \mathbb{R}^{n}$ \\
\hline$\left(x_{i}, y_{i}\right)$ & Global coordinates of CM of link $i$. & $x, y \in \mathbb{R}^{n}$ \\
\hline$\left(p_{x}, p_{y}\right)$ & $\begin{array}{l}\text { Global coordinates of CM of snake } \\
\text { robot. }\end{array}$ & $p \in \mathbb{R}^{2}$ \\
\hline$u_{i}$ & $\begin{array}{l}\text { Actuator torque exerted on link } i \text { from } \\
\text { link } i+1 \text {. }\end{array}$ & $u \in \mathbb{R}^{n-1}$ \\
\hline$u_{i-1}$ & $\begin{array}{l}\text { Actuator torque exerted on link } i \text { from } \\
\text { link } i-1 \text {. }\end{array}$ & $u \in \mathbb{R}^{n-1}$ \\
\hline$f_{R, x, i}$ & Friction force on link $i$ in $x$ direction. & $f_{R, x} \in \mathbb{R}^{n}$ \\
\hline$f_{R, y, i}$ & Friction force on link $i$ in $y$ direction. & $f_{R, y} \in \mathbb{R}^{n}$ \\
\hline$h_{x, i}$ & $\begin{array}{l}\text { Joint constraint force in } x \text { direction on } \\
\text { link } i \text { from link } i+1 .\end{array}$ & $h_{x} \in \mathbb{R}^{n-1}$ \\
\hline$h_{y, i}$ & $\begin{array}{l}\text { Joint constraint force in } y \text { direction on } \\
\text { link } i \text { from link } i+1 .\end{array}$ & $h_{y} \in \mathbb{R}^{n-1}$ \\
\hline$h_{x, i-1}$ & $\begin{array}{l}\text { Joint constraint force in } x \text { direction on } \\
\text { link } i \text { from link } i-1 \text {. }\end{array}$ & $h_{x} \in \mathbb{R}^{n-1}$ \\
\hline$h_{y, i-1}$ & $\begin{array}{l}\text { Joint constraint force in } y \text { direction on } \\
\text { link } i \text { from link } i-1 \text {. }\end{array}$ & $h_{y} \in \mathbb{R}^{n-1}$ \\
\hline
\end{tabular}

$$
\text { TABLE I }
$$

DEFINED MATHEMATICAL IDENTITIES. $\sin \theta:=\left[\sin \theta_{1} \ldots \sin \theta_{n}\right]^{T} \in \mathbb{R}^{n} \cos \theta:=\left[\cos \theta_{1} . . \cos \theta_{n}\right]^{T} \in \mathbb{R}^{n}$ $S_{\theta}:=\operatorname{diag}\left(\sin \theta_{1}, . ., \sin \theta_{n}\right) \quad C_{\theta}:=\operatorname{diag}\left(\cos \theta_{1}, . ., \cos \theta_{n}\right)$

\section{B. Kinematics}

The snake robot moves in the horizontal plane and has a total of $n+2$ degrees of freedom. The absolute angle, $\theta_{i}$, of link $i$ is expressed with respect to the global $x$ axis with counterclockwise positive direction. As seen in Fig. 2, the relative angle between link $i$ and link $i+1$ is given by $\phi_{i}=\theta_{i}-\theta_{i+1}$. The local coordinate system of each link is fixed in the CM (center of mass) of the link with $x$ (tangential) and $y$ (normal) axis oriented such that they are oriented in the directions of the global $x$ and $y$ axis, respectively, when the link angle is zero. The rotation matrix from the global frame to the frame of link $i$ is given by

$$
R_{\text {link }, i}^{\text {global }}=\left[\begin{array}{cc}
\cos \theta_{i} & -\sin \theta_{i} \\
\sin \theta_{i} & \cos \theta_{i}
\end{array}\right]
$$

The position of the snake robot, $p$, is described through the coordinates of its CM (center of mass) and is given by

$$
p:=\left[\begin{array}{l}
p_{x} \\
p_{y}
\end{array}\right]=\left[\begin{array}{l}
\frac{1}{n m} \sum_{i=1}^{n} m x_{i} \\
\frac{1}{n m} \sum_{i=1}^{n} m y_{i}
\end{array}\right]=\frac{1}{n}\left[\begin{array}{l}
e^{T} x \\
e^{T} y
\end{array}\right]
$$

It is shown in [10] that the position of the CM of each link along the global $x$ and $y$ axis, respectively, is given by

$$
\begin{gathered}
x=-l N^{T} \cos \theta+e p_{x} \\
y=-l N^{T} \sin \theta+e p_{y} \\
N:=A^{T}\left(D D^{T}\right)^{-1} D \in \mathbb{R}^{n \times n}
\end{gathered}
$$

The linear velocities of the links are derived by differentiating (10) with respect to time. This gives

$$
\begin{aligned}
& \dot{x}=l N^{T} S_{\theta} \dot{\theta}+e \dot{p}_{x} \\
& \dot{y}=-l N^{T} C_{\theta} \dot{\theta}+e \dot{p}_{y}
\end{aligned}
$$

\section{Viscous friction model}

We have chosen to employ a viscous ground friction model in this study. Alternatively, we could have used a Coulomb friction model. However, we conjecture that a viscous and a Coulomb friction model are very similar from a control perspective when the friction is non-uniform. The work in e.g. [5] supports this conjecture. Moreover, viscous friction leads to much simpler equations compared to Coulomb friction. This greatly simplifies the analysis in Section IV.

Under non-uniform friction conditions, a link has two viscous friction coefficients, $c_{t}$ and $c_{n}$, describing the friction force in the tangential (along link $x$ axis) and normal (along link $y$ axis) direction of the link, respectively. Using (8), the friction force on link $i$ in the global frame as a function of the global link velocity, $\dot{x}_{i}$ and $\dot{y}_{i}$, is given by

$$
\begin{aligned}
f_{R, i}^{\text {global }} & =R_{\text {link }, i}^{\text {global }} f_{R, i}^{\text {link }, i}=-R_{\text {link }, i}^{\text {global }}\left[\begin{array}{cc}
c_{t} & 0 \\
0 & c_{n}
\end{array}\right] v_{i}^{\text {link }, i} \\
& =-R_{\text {link }, i}^{\text {global }}\left[\begin{array}{cc}
c_{t} & 0 \\
0 & c_{n}
\end{array}\right]\left(R_{\text {link }, i}^{\text {global }}\right)^{T}\left[\begin{array}{c}
\dot{x}_{i} \\
\dot{y}_{i}
\end{array}\right]
\end{aligned}
$$

where $f_{R, i}^{\text {link, } i}$ and $v_{i}^{\text {link, } i}$ are, respectively, the friction force and the link velocity expressed in the local link frame. 
Performing the matrix multiplication and assembling the friction forces on all links in matrix form gives

$$
f_{R}=-\left[\begin{array}{cc}
c_{t}\left(C_{\theta}\right)^{2}+c_{n}\left(S_{\theta}\right)^{2} & \left(c_{t}-c_{n}\right) S_{\theta} C_{\theta} \\
\left(c_{t}-c_{n}\right) S_{\theta} C_{\theta} & c_{t}\left(S_{\theta}\right)^{2}+c_{n}\left(C_{\theta}\right)^{2}
\end{array}\right]\left[\begin{array}{l}
\dot{x} \\
\dot{y}
\end{array}\right]
$$

where $f_{R}=\left[\begin{array}{ll}f_{R, x}^{T} & f_{R, y}^{T}\end{array}\right]^{T} \in \mathbb{R}^{2 n}$.

\section{Equations of motion}

This section presents the equations of motion of the snake robot in terms of the acceleration of the link angles, $\ddot{\theta}$, and the acceleration of the $\mathrm{CM}$ of the snake robot, $\ddot{p}$. These coordinates describe all $n+2$ DOFs of the snake robot.

The forces and torques acting on link $i$ are visualized in Fig. 3. The force balance for link $i$ in global frame coordinates is given by

$$
\begin{aligned}
& m \ddot{x}_{i}=f_{R, x, i}+h_{x, i}-h_{x, i-1} \\
& m \ddot{y}_{i}=f_{R, y, i}+h_{y, i}-h_{y, i-1}
\end{aligned}
$$

while the torque balance for link $i$ is given by

$$
\begin{gathered}
\ddot{\theta}_{i}=u_{i}-u_{i-1} \\
-l \sin \theta_{i}\left(h_{x, i}+h_{x, i-1}\right)+l \cos \theta_{i}\left(h_{y, i}+h_{y, i-1}\right)
\end{gathered}
$$

Through straightforward calculations, it is shown in [10] that (15) and (16) may be rewritten for all links and combined into the following complete model of the snake robot:

$$
\begin{aligned}
M \ddot{\theta} & =W \dot{\theta}^{2}+l S_{\theta} N f_{R, x}-l C_{\theta} N f_{R, y}+D^{T} u \\
n m \ddot{p} & =n m\left[\begin{array}{l}
\ddot{p}_{x} \\
\ddot{p}_{y}
\end{array}\right]=E^{T} f_{R}=\left[\begin{array}{l}
e^{T} f_{R, x} \\
e^{T} f_{R, y}
\end{array}\right]
\end{aligned}
$$

where $\theta$ and $p$ represent the $n+2$ generalized coordinates of the system, $\dot{\theta}^{2}=\operatorname{diag}(\dot{\theta}) \dot{\theta}$, and

$$
\begin{gathered}
M:=J I_{n \times n}+m l^{2}\left(S_{\theta} V S_{\theta}+C_{\theta} V C_{\theta}\right) \\
W:=m l^{2}\left(C_{\theta} V S_{\theta}-S_{\theta} V C_{\theta}\right) \\
N:=A^{T}\left(D D^{T}\right)^{-1} D \\
V:=A^{T}\left(D D^{T}\right)^{-1} A
\end{gathered}
$$

The model may be written more compactly by introducing the state variable $x=\left[\begin{array}{llll}\theta^{T} & p^{T} & \dot{\theta}^{T} & \dot{p}^{T}\end{array}\right]^{T} \in \mathbb{R}^{2 n+4}$ and combining (17) and (18) into the single equation

$$
\dot{x}=f(x, t)
$$

where we have assumed that $u=u(x, t)$. See [13] for a detailed outline of (20).

\section{ANALYSIS AND EXPLANATION OF SNAKE LOCOMOTION}

This section presents an analysis of snake locomotion that explains how non-uniform viscous ground friction conditions enable snake robots to locomote forward on a planar surface.

\section{A. Analysis of propulsive forces during snake locomotion}

Non-uniform friction generally means that the friction coefficients in the tangential and normal direction of the links are different $\left(c_{t} \neq c_{n}\right)$. However, we will only focus on the commonly assumed property of snake robots that the normal direction friction is larger than the tangential friction $\left(c_{t}<c_{n}\right)$.

We first derive an expression for the total force propelling the CM (center of mass) of the snake robot forward as a function of the linear link velocities. We will call this the propulsive force on the snake robot and denote it by $F_{\text {prop }}$. The forward direction of motion is assumed to be along the global positive $x$ axis. As described in Section (III-B), the angle $\theta_{i}$ of link $i$ is expressed with respect to the global $x$ axis with counterclockwise positive direction. The propulsive force is simply the sum of all external forces on the snake robot in the global $x$ direction and is given from (18) as

$$
F_{\text {prop }}=n m \ddot{p}_{x}=e^{T} f_{R, x}
$$

Inserting $f_{R, x}$ from (14) into (21) gives

$$
F_{\text {prop }}=-e^{T}\left(\left(c_{t}\left(C_{\theta}\right)^{2}+c_{n}\left(S_{\theta}\right)^{2}\right) \dot{x}+\left(c_{t}-c_{n}\right) S_{\theta} C_{\theta} \dot{y}\right)
$$

The purpose of the vector $e^{T}$ is to sum up the friction force contributions from all the links. We may therefore write (22) as a summation in order to investigate the propulsive force contribution from a single link.

$$
\begin{aligned}
F_{\text {prop }}= & -\sum_{i=1}^{n}\left(\left(c_{t} \cos ^{2} \theta_{i}+c_{n} \sin ^{2} \theta_{i}\right) \dot{x}_{i}\right. \\
& \left.+\left(c_{t}-c_{n}\right) \sin \theta_{i} \cos \theta_{i} \dot{y}_{i}\right)
\end{aligned}
$$

The propulsive force from a single link, $F_{\mathrm{prop}, i}$, is in other words given by

$$
F_{\text {prop }, i}=-F_{x}\left(\theta_{i}\right) \dot{x}_{i}-F_{y}\left(\theta_{i}\right) \dot{y}_{i}
$$

where

$$
\begin{aligned}
& F_{x}\left(\theta_{i}\right):=c_{t} \cos ^{2} \theta_{i}+c_{n} \sin ^{2} \theta_{i} \\
& F_{y}\left(\theta_{i}\right):=\left(c_{t}-c_{n}\right) \sin \theta_{i} \cos \theta_{i}
\end{aligned}
$$

We see from (24) that $F_{\text {prop }, i}$ consists of two components, i.e. one involving the linear velocity of the link in the forward direction of motion, $F_{x}\left(\theta_{i}\right) \dot{x}_{i}$, and one involving the linear velocity normal to the direction of motion, $F_{y}\left(\theta_{i}\right) \dot{y}_{i}$. Due to the minus signs in (24), the products $F_{x}\left(\theta_{i}\right) \dot{x}_{i}$ and $F_{y}\left(\theta_{i}\right) \dot{y}_{i}$ provide a positive contribution to the propulsive force only if they are negative. Since the viscous friction coefficients, $c_{t}$ and $c_{n}$, are always positive, the expression $F_{x}\left(\theta_{i}\right)$ is obviously always positive. We assume that the snake robot is not generating waves that involve $x$ direction velocities of any of the links opposite to the direction of motion. When the snake robot is moving in the forward direction $\left(\dot{p}_{x}>0\right)$, we therefore have that $\dot{x}_{i}>0$, which means that the product $F_{x}\left(\theta_{i}\right) \dot{x}_{i}$ of the propulsive force is always positive. This product is therefore not contributing to the forward propulsion of the robot, but rather opposing it. This is also expected since the snake robot must naturally be subjected to a friction force in the opposite direction of the motion.

Any maintained propulsive force in the forward direction of motion must therefore be produced by the sideways motion of the links, i.e. the product $F_{y}\left(\theta_{i}\right) \dot{y}_{i}$. A plot of $F_{y}\left(\theta_{i}\right)$ for different values of the normal friction coefficient $c_{n}$, while keeping the tangential friction coefficient $c_{t}$ fixed, is shown in Fig. 4. For each plot, the angle between the link and the forward direction, $\theta_{i}$, is varied from $-90^{\circ}$ to $90^{\circ}$.

The sideways motion of the links have no effect on the propulsive force on the snake robot when the friction coefficients are equal since this gives $F_{y}\left(\theta_{i}\right)=0$. However, when $c_{n}>c_{t}$, Fig. (4) reveals that $F_{y}\left(\theta_{i}\right)$ is negative as long as $\theta_{i}$ is positive, and vice versa. This means that the product $F_{y}\left(\theta_{i}\right) \dot{y}_{i}$ is negative as long as $\operatorname{sgn}\left(\theta_{i}\right)=\operatorname{sgn}\left(\dot{y}_{i}\right)$. The sideways motion of a link is in other words contributing to the propulsion of the snake robot as long as $\theta_{i}$ is positive 


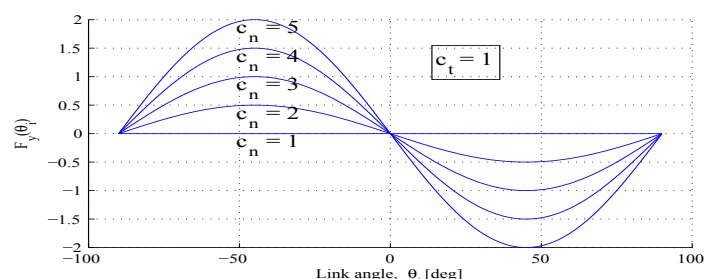

Fig. 4. The mapping from sideways link motion to forward propulsion for different viscous friction coefficients.

during leftward motion of the link (left with respect to the direction of motion) and negative during rightward motion of the link (right with respect to the direction of motion). This fundamental relationship may be written $\operatorname{sgn}\left(F_{\text {prop }, i}\right)=$ $\operatorname{sgn}\left(\operatorname{sgn}\left(\theta_{i}\right) \operatorname{sgn}\left(\dot{y}_{i}\right)\right)$.

It is straightforward to calculate that the extrema of $F_{y}\left(\theta_{i}\right)$ occur at $\theta_{i}= \pm 45^{\circ}$. This is also seen from Fig. 4. This means that, for a given $\dot{y}_{i}$, a link produces its highest propulsive force when it forms an angle of $\pm 45^{\circ}$ with the forward direction of motion. It is also evident from (24) that the magnitude of $F_{y}\left(\theta_{i}\right) \dot{y}_{i}$, and thereby the magnitude of the propulsive force, $\left|F_{\text {prop }, i}\right|$, is increased by increasing $c_{n}$ with respect to $c_{t}$, or by increasing the magnitude of the sideways link velocity, $\left|\dot{y}_{i}\right|$.

It should now be clear that the function $F_{y}\left(\theta_{i}\right)$ maps the link velocities normal to the direction of motion into force components in the direction of motion. The following simple analogy may help understand this result. Imagine a small, hand-held, wheeled wagon of some sort. The direction of the wheels corresponds to the tangential direction of a snake robot link. Obviously, the friction coefficient of the wagon in the direction of the wheels is smaller than the friction coefficient normal to the wheels. Now assume that you push the wagon across a table in the direction of the wheels. While maintaining constant direction of motion, assume that you slowly rotate the wagon about the vertical axis, thereby forcing the wheels to slip. The hand that push and rotate the wagon will now feel a tendency of the wagon to move sideways in the same direction towards which the wagon was rotated. This is in accordance with the results presented above. The above analysis proves the following propositions:

Proposition 1: A snake robot with non-uniform friction properties on a flat surface achieves forward propulsion through the sideways velocity components of its links (sideways with respect to the forward direction).

Proposition 2: The direction of the propulsive force generated by the sideways motion of link $i$ is given by the fundamental relationship $\operatorname{sgn}\left(F_{\text {prop }, i}\right)=\operatorname{sgn}\left(\operatorname{sgn}\left(\theta_{i}\right) \operatorname{sgn}\left(\dot{y}_{i}\right)\right)$.

Proposition 3: The function $F_{y}\left(\theta_{i}\right)$ maps the link velocities normal to the direction of motion into force components in the direction of motion.

Proposition 4: The magnitude of the propulsive force generated by link $i,\left|F_{\text {prop }, i}\right|$, is increased by increasing $c_{n}$ with respect to $c_{t}$, or by increasing the magnitude of the sideways link velocity, $\left|\dot{y}_{i}\right|$.

Proposition 5: For a given $\dot{y}_{i}$, a link produces its highest propulsive force when it forms an angle of $\theta_{i}= \pm 45^{\circ}$ with the forward direction of motion.

Note that these results are general in the sense that no assumptions have been made regarding the actual motion pattern displayed by the snake robot.

\section{B. Synthesis of propulsive motion for the snake robot}

The analysis from the previous section enables us to deduce how the snake robot links should be moved in order to propel the snake robot forward along the positive $x$ axis. The following analysis focusses on manipulating the magnitude, $\left|F_{\mathrm{prop}, i}\right|$, and direction, $\operatorname{sgn}\left(F_{\mathrm{prop}, i}\right)$, of the propulsive force from each link.

From Proposition 1, we know that propulsive forces are generated by moving the links in the normal direction with respect to the desired direction of motion. We require the amplitude of this normal direction link motion to be bounded since the snake robot otherwise would diverge from the desired path. We therefore conclude that the links must have a periodic velocity component normal to the direction of motion. This suggests that each link should be moved alternatingly to the left and right with respect to the direction of motion, and may be achieved by letting the trajectory of each link angle have the form

$$
\theta_{i}=\alpha \sin (\omega t) \quad, i=\{1, . ., n\}
$$

where $\alpha>0$ is the maximum amplitude of the link angles during the locomotion, $\omega>0$ is the angular frequency of the periodic motion, and $t$ denotes time. For simplicity, we assume that $\alpha$ and $\omega$ are constant and identical for all links. A recent result in [13] shows that the joint angles of a snake robot during locomotion should be out of phase since this improves the controllability properties of the snake robot. This suggests that (26) should be modified to

$$
\theta_{i}=\alpha \sin (\omega t+(i-1) \delta) \quad, i=\{1, . ., n\}
$$

where $\delta$ is the phase shift between adjacent links. For simplicity, we assume a constant phase shift between the links.

We now investigate how $\alpha, \omega$, and $\delta$ affect $\left|F_{\text {prop }, i}\right|$ and $\operatorname{sgn}\left(F_{\text {prop }, i}\right)$ as the snake robot moves along the global $x$ axis. To simplify the analysis, we assume that the snake robot consists of only $n=3$ links. This is the minimum number of links required to achieve propulsion since phase shift between joints requires at least two joints. The below analysis for $n=3$ links also apply to robots with $n>3$ links since a snake robot can be regarded as a connection of multiple three-linked segments. The trajectories of the link angles are given from (27) as

$$
\begin{gathered}
\theta_{1}=\alpha \sin (\omega t) \\
\theta_{2}=\alpha \sin (\omega t+\delta) \\
\theta_{3}=\alpha \sin (\omega t+2 \delta)
\end{gathered}
$$

which, when differentiated with respect to time, gives the following angular link velocitites:

$$
\begin{gathered}
\dot{\theta}_{1}=\alpha \omega \cos (\omega t) \\
\dot{\theta}_{2}=\alpha \omega \cos (\omega t+\delta) \\
\dot{\theta}_{3}=\alpha \omega \cos (\omega t+2 \delta)
\end{gathered}
$$

The normal direction velocity of each link is given by (12). We disregard the normal direction velocity of the $\mathrm{CM}$ of 


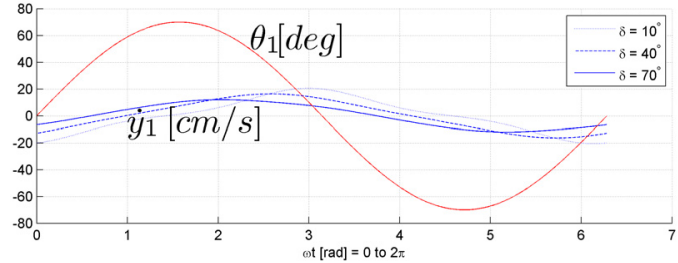

Fig. 5. The relation between $\theta_{1}$ and $\dot{y}_{1}$ for $\alpha=70^{\circ}, \omega=70^{\circ}$, and $\delta=10^{\circ}$ (dotted), $40^{\circ}$ (dashed), $70^{\circ}$ (solid).

the snake robot by setting $\dot{p}_{y} \approx 0$. This is a fairly accurate approximation as long as the snake robot is moving along the global $x$ axis, which is the case for this analysis. Inserting (28) and (29) into (12) gives

$$
\begin{gathered}
\dot{y}_{1}=-\frac{\alpha \omega l}{3}(2 \cos (\omega t) \cos (\alpha \sin (\omega t))) \\
-\frac{\alpha \omega l}{3}(3 \cos (\omega t+\delta) \cos (\alpha \sin (\omega t+\delta))) \\
-\frac{\alpha \omega l}{3}(\cos (\omega t+2 \delta) \cos (\alpha \sin (\omega t+2 \delta))) \\
\dot{y}_{2}=\frac{\alpha \omega l}{3}(\cos (\omega t) \cos (\alpha \sin (\omega t))) \\
-\frac{\alpha \omega l}{3}(\cos (\omega t+2 \delta) \cos (\alpha \sin (\omega t+2 \delta))) \\
+\frac{\alpha \omega l}{3}(3 \cos (\omega t+\delta) \cos (\alpha \sin (\omega t+\delta))) \\
+\frac{\alpha \omega l}{3}(2 \cos (\omega t+2 \delta) \cos (\alpha \sin (\omega t+2 \delta)))
\end{gathered}
$$

Proposition 4 tells us that $\left|F_{\text {prop }, i}\right|$ is increased by increasing $\left|\dot{y}_{i}\right|$. From (30)-(32), it is therefore clear that $\left|F_{\text {prop }, i}\right|$ is increased by increasing $\alpha$ and/or $\omega$. We now determine if $\delta$ should be positive or negative in order to achieve $\operatorname{sgn}\left(F_{\text {prop }, i}\right)=1$, which is necessary to propel the snake robot forward along the global $x$ axis. From Proposition 2, we know that $\operatorname{sgn}\left(F_{\text {prop }, i}\right)=1$ requires $\operatorname{sgn}\left(\theta_{i}\right)=\operatorname{sgn}\left(\dot{y}_{i}\right)$. Considering $\dot{y}_{2}$ in (31) (since this expression is easy to analyze), it is seen through pure inspection that $\dot{y}_{2}=0$ when $\omega t=-\delta$. When $\omega t=-\delta$, we see from (28) and (29) that $\theta_{2}=0$ and $\dot{\theta}_{2}=\alpha \omega>0 . \theta_{2}$ is in other words about to become positive, which means that we also require $\dot{y}_{2}$ to become positive. This is the case if $\ddot{y}_{2}>0$ when $\omega t=-\delta$. Differentiating (31) with respect to time gives

$$
\begin{gathered}
\left.\ddot{y}_{2}\right|_{\omega t=}=-\delta=\frac{2 \alpha^{2} \omega^{2} l}{3} \cos ^{2}(\delta) \sin (\alpha \sin (\delta)) \\
+\frac{2 \alpha \omega^{2} l}{3} \sin (\delta) \cos (\alpha \sin (\delta))
\end{gathered}
$$

from which it is easily seen that $\ddot{y}_{2}>0$ when $\delta>0$, i.e. $\operatorname{sgn}\left(F_{\text {prop }, i}\right)=1$ when $\delta>0$. This indicates that the links generate positive propulsive forces if $\delta>0$.

In order to verify that forward propulsion requires $\delta>$ 0 , we have plotted (28) and (30)-(32) together in Fig. 5-7 for $\alpha=70^{\circ}, \omega=70^{\circ}$, and for different positive values of $\delta$ over a period of $\omega t$ from 0 to $2 \pi$. The figures show that $\operatorname{sgn}\left(F_{\text {prop }, i}\right)=\operatorname{sgn}\left(\operatorname{sgn}\left(\theta_{i}\right) \operatorname{sgn}\left(\dot{y}_{i}\right)\right)=1$ is always satisfied for link 2 , but only satisfied over about half the period for link 1 and 3 when $\delta$ is small. As $\delta$ is increased, $\operatorname{sgn}\left(F_{\text {prop }, i}\right)=1$ is satisfied over a larger portion of the period. We do not attempt to determine the optimal choice of $\delta$ in this analysis, but conclude that positive propulsive forces requires $\delta>0$.

The above analysis proves the following propositions:

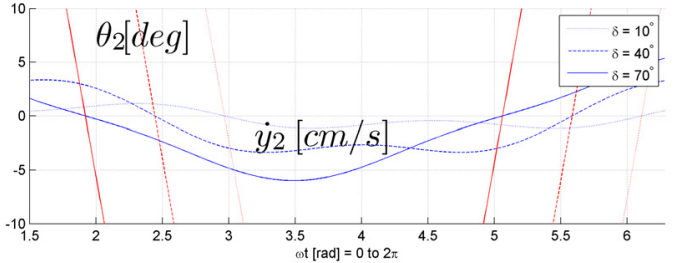

Fig. 6. The relation between $\theta_{2}$ and $\dot{y}_{2}$ for $\alpha=70^{\circ}, \omega=70^{\circ}$, and $\delta=10^{\circ}$ (dotted), $40^{\circ}$ (dashed), $70^{\circ}$ (solid). The plot is zoomed in.

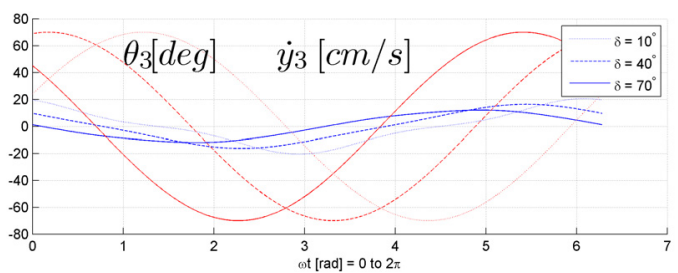

Fig. 7. The relation between $\theta_{3}$ and $\dot{y}_{3}$ for $\alpha=70^{\circ}, \omega=70^{\circ}$, and $\delta=10^{\circ}$ (dotted), $40^{\circ}$ (dashed), $70^{\circ}$ (solid).

Proposition 6: A snake robot with non-uniform friction properties on a flat surface achieves forward propulsion by moving its links according to $\theta_{i}=\alpha \sin (\omega t+(i-1) \delta)$ where $i=\{1, . ., n\}, \alpha>0, \omega>0$, and $\delta>0$.

Proposition 7: The magnitude of the propulsive force generated by link $i,\left|F_{\text {prop }, i}\right|$, is increased by increasing $\alpha$ and/or $\omega$.

Note that the expression for the link angle trajectories in (27) is in accordance with previous work on snake robots by Hirose [2], where the required motion of the links was deduced from empirical studies of biological snakes. The choices made in the above study are obviously inspired by the work in [2]. However, an important purpose of this study is to show that it is possible to develop logical arguments merely from an analysis of the equations of motion of a snake robot and thereby arrive at similar conclusions as Hirose.

\section{Controller Design}

This section proposes a controller that will enable the snake robot to locomote forward along the global positive $x$ axis. The analysis in Section IV-B suggests that this is achieved by generating sinusoidal motion of the absolute link angles while maintaining a positive phase shift $\delta$ between adjacent links according to (27). However, since the snake robot is underactuated with only $n-1$ control inputs, it is not possible to control all $n$ link angles independently. We therefore choose to control the $n-1$ relative joint angles (denoted by $\phi$ as described in Section III-B) in order to generate a phase shifted sinusoidal motion of the $n$ absolute link angles, and introduce a joint angle offset in order to control the absolute heading of the snake robot. The reference motion of $\phi$ is therefore given by

$$
\phi_{i, \mathrm{ref}}=\alpha \sin (\omega t+(i-1) \delta)+\phi_{\mathrm{offset}}
$$

where $\alpha, \omega$, and $\delta$ were defined in Section IV-B, $\phi_{\text {offset }}$ is the joint angle offset, and $i=\{1, . ., n-1\}$. The offset is identical for all joints and affects the direction of the motion 


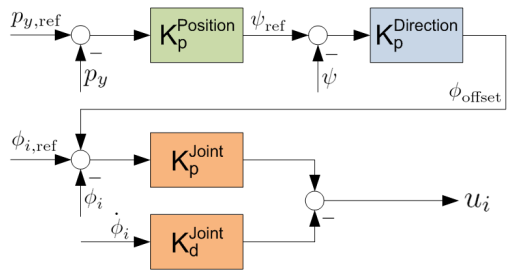

Fig. 8. The controller of the snake robot.

by making the link motion asymmetrical with respect to the current heading of the robot. This joint angle reference trajectory was first introduced in [2].

We denote the absolute heading of the snake robot by $\psi$ and calculate it as the mean of all the link angles:

$$
\psi=\frac{1}{n} \sum_{i=1}^{n} \theta_{i} \quad, i=\{1, . ., n\}
$$

We use the joint angle offset $\phi_{\text {offset }}$ to control the heading $\psi$ of the robot by employing the simple proportional controller

$$
\phi_{\text {offset }}=K_{p}^{\text {Direction }}\left(\psi_{\text {ref }}-\psi\right)
$$

where $K_{p}^{\text {Direction }}$ is the controller gain and $\psi_{\text {ref }}$ is the reference heading. We use the reference heading $\psi_{\text {ref }}$ to control the $y$ direction position, $p_{y}$, of the robot by employing the additional proportional controller

$$
\psi_{\text {ref }}=K_{p}^{\text {Position }}\left(p_{y, \text { ref }}-p_{y}\right)
$$

where $K_{p}^{\text {Position }}$ is the controller gain and $p_{y \text {,ref }}$ is the reference $y$ direction position. Since the goal is locomotion along the global $x$ axis, we set $p_{y \text {,ref }}=0$. The reason for not including integral action in the above controllers is that directional control will not work if $\phi_{\text {offset }}$ becomes too large. We use a PD-controller to control the relative joint angles of the snake robot according to

$$
u_{i}=K_{p}^{\mathrm{Joint}}\left(\phi_{i, \mathrm{ref}}-\phi_{i}\right)-K_{d}^{\mathrm{Joint}} \dot{\phi}_{i}
$$

where $i=\{1, . ., n-1\}$ and where we have chosen to set $\dot{\phi}_{i, \text { ref }}=0$ since the purpose of the derivative part of the controller is simply to damp the motion if the velocities become large. The complete controller for the snake robot is depicted in Fig. 8.

\section{STABILITY ANALYSis BASED ON THE PoINCARÉ MAP}

This section employes the theory of Poincaré maps, presented in Section II, to prove that the controller in (38) generates a locally exponentially stable periodic orbit in the state space of the snake robot.

\section{A. Converting the snake robot model to a time-periodic autonomous system}

During locomotion along the global positive $x$ axis, our goal is that the $x$ axis position of the snake robot, $p_{x}$, increases, while all other states of the snake robot in (20) trace out a stable limit cycle in the state space. We therefore exclude $p_{x}$ from the Poincare map of the snake robot. This corresponds to a partial Poincaré map [12]. Exclusion of $p_{x}$ has no effect on the other state variables since $p_{x}$ is not present in any of their derivatives in (20). The analysis of snake locomotion in Section IV, which is the basis of the controller in (38), enables us to argue that forward motion along the $x$ axis (increase of $p_{x}$ ) is achieved as long as the remaining state variables trace out a stable periodic orbit.

Stability analysis of the time-periodic state variables of the snake robot by use of Poincaré maps requires the model of the snake robot in (20) to represent an autonomous system, i.e. a system not explicitly dependent on time. The controller in (38), however, makes the system non-autonomous since time $t$ is present in the expressions for the joint torque inputs. We therefore follow the approach described in [11] in order to convert the snake robot model to an autonomous system by simply augmenting the state vector $x$ with an extra state $\beta=2 \pi t / T$, where $T=2 \pi / \omega$ is the period of the cyclic locomotion generated by the controller in (38). We make $\beta$ periodic by enforcing that $0 \leq \beta<2 \pi$, i.e. we set $\beta$ to zero each time $\beta=2 \pi$. The model (20) with the controller (38) therefore represents the following autonomous system:

$$
\begin{array}{cc}
\dot{x}=f\left(x, \frac{T}{2 \pi} \beta\right) & , \quad x\left(t_{0}\right)=x_{0} \\
\dot{\beta}=\frac{2 \pi}{T} & , \quad \beta\left(t_{0}\right)=\frac{2 \pi t_{0}}{T}
\end{array}
$$

We have, in other words, encapsulated time $t$ in the new state variable $\beta$, which is periodic since $0 \leq \beta<2 \pi$.

\section{B. Specification of the Poincaré section for the snake robot}

We choose the global $x$ axis as the Poincaré section $\mathcal{S}$ of the system in (39). Since $p_{x}$ is not included in the Poincare map, we write $\mathcal{S}=\left\{\left(\theta, p_{y}, \dot{\theta}, \dot{p}, \beta\right) \mid p_{y}=0\right\}$. Following the notation in Section II, the vector of independent timeperiodic states constrained to $\mathcal{S}$ is given by

$$
\bar{x}=\left[\begin{array}{llll}
\theta^{T} & \dot{\theta}^{T} & \dot{p}^{T} & \beta
\end{array}\right]^{T} \in \mathbb{R}^{2 n+3}
$$

\section{Simulation and stability analysis}

We consider a three-linked snake robot where $n=3, l=$ $0.07 \mathrm{~m}, m=1 \mathrm{~kg}$, and $J=0.0016 \mathrm{kgm}^{2}$. The parameters of the controller in (38) were (based on trial-and-error) set to $\alpha=70^{\circ}, \omega=70^{\circ} / s, \delta=70^{\circ}, K_{p}^{\text {Joint }}=20, K_{d}^{\text {Joint }}=5$, $K_{p}^{\text {Direction }}=2$, and $K_{p}^{\text {Position }}=5$. The model in (39) was simulated using Matlab R2008a on a laptop running Windows $X P$. The ode45 solver in Matlab was used with a relative and absolute error tolerance of $10^{-6}$.

The Poincaré map of the snake robot model in (39) was calculated as described in Section II-B.1. The NewtonRaphson algorithm described in Section II-B.2 calculated the fixed point, $\bar{x}^{*} \in \mathbb{R}^{9}$, of the Poincaré map as

$$
\begin{aligned}
& \bar{x}^{*}=\left[\begin{array}{llll}
-13.6^{\circ} & -34.5^{\circ} & 26.3^{\circ} & -81.2^{\circ} / \mathrm{s}
\end{array}\right. \\
& \left.10.0^{\circ} / \mathrm{s} \quad 89.3^{\circ} / \mathrm{s} \quad 5.1^{\mathrm{cm}} / \mathrm{s} \quad-1.6^{\mathrm{cm}} / \mathrm{s} \quad 170^{\circ}\right]^{T}
\end{aligned}
$$

A plot of the cyclic locomotion of the snake robot over one period is shown in Fig. 9. The initial state of the snake robot is given by $\bar{x}^{*}$. The initial position is $p=0$. After one period of the motion, the state variables return to their initial value, $\bar{x}^{*}$. At this point, however, the position of the snake robot along the $x$ axis has increased, which was also our goal. To clearly illustrate the limit cycle behaviour of the periodic state variables in (40), a 3D plot of the three absolute link angles over one period is given in Fig. 10 .

The Jacobian linearization of the Poincare map about the fixed point (41) were calculated as described in Section IIB.3. The eigenvalues the Jacobian matrix $J_{P}\left(\bar{x}^{*}\right) \in \mathbb{R}^{9 \times 9}$ were given by 

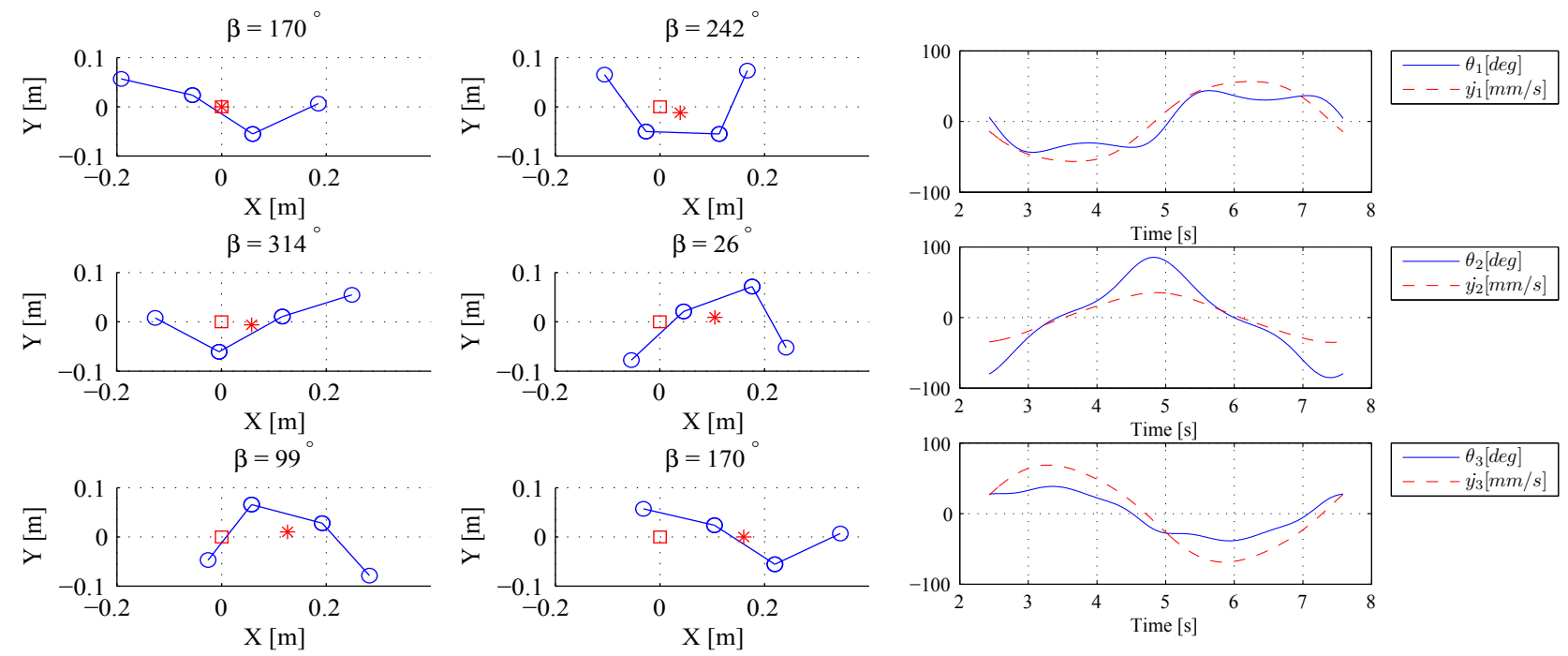

Fig. 9. The motion of the snake robot over one period of the cyclic locomotion.

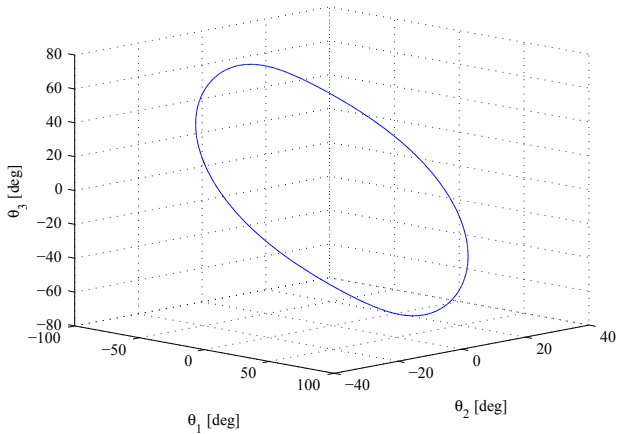

Fig. 10. The limit cycle traced out by the link angles of the snake robot.

$$
\begin{gathered}
\operatorname{eig}\left(J_{P}\left(\bar{x}^{*}\right)\right)=\left[\begin{array}{lll}
0.33 & 0.10 & 0.0035 \\
-0.0026 & -9.3 \times 10^{-4} & 5.4 \times 10^{-5} \\
-3.1 \times 10^{-5} & -5.5 \times 10^{-8} & 3.6 \times 10^{-6}
\end{array}\right]
\end{gathered}
$$

The magnitude of all the eigenvalues are strictly less than one. The periodic orbit traced out by the variables in (40) is therefore locally exponentially stable. All initial conditions inside the basin of attraction of this periodic orbit will converge exponentially to this periodic orbit. Identifying the basin of attraction for $\bar{x}^{*}$ is a topic of future research.

We have now proven that the controller in (38) generates a stable periodic orbit comprising all state variables, except the position $p_{x}$. Based on the analysis in Section IV, this implies that the snake robot is locomoting forward. In particular, Proposition 2 in Section IV-A states that the direction of the propulsive force on the snake robot from link $i$ is given by $\operatorname{sgn}\left(F_{\text {prop }, i}\right)=\operatorname{sgn}\left(\operatorname{sgn}\left(\theta_{i}\right) \operatorname{sgn}\left(\dot{y}_{i}\right)\right)$. A plot of $\theta_{i}$ and $\dot{y}_{i}$ $(i=1,2,3)$ over one period is given in Fig. 11, which clearly shows that $\operatorname{sgn}\left(F_{\text {prop }, i}\right)=1$ over the majority of the period. This means that the net propulsive force on the snake robot is positive.

\section{CONCLUSION}

This paper has explained how non-uniform viscous ground friction conditions enable snake robots to locomote
Fig. 11. Plot of $\theta_{i}$ and $\dot{y}_{i}(i=1,2,3)$ over one period of the cyclic locomotion.

forward on a planar surface. The explanation is based on a simple mapping from link velocities normal to the direction of motion into propulsive forces in the direction of motion. The paper has also proposed a controller for propelling a snake robot along the global $x$ axis. We applied the method of Poincaré maps to prove that the remaining state variables of the robot traced out an exponentially stable periodic orbit, which in turn implied that the snake robot is locomoting in the desired forward direction.

\section{REFERENCES}

[1] J. Gray, "The mechanism of locomotion in snakes," J. Exp. Biol., vol. 23, no. 2, pp. 101-120, 1946.

[2] S. Hirose, Biologically Inspired Robots: Snake-Like Locomotors and Manipulators. Oxford: Oxford University Press, 1993.

[3] T. Kane and D. Lecison, "Locomotion of snakes: A mechanical 'explanation'," Int. J. Solids Struct., vol. 37, no. 41, pp. 5829-5837, October 2000.

[4] S. Ma, "Analysis of creeping locomotion of a snake-like robot," $A d v$. Robotics, vol. 15, no. 2, pp. 205-224, 2001.

[5] M. Saito, M. Fukaya, and T. Iwasaki, "Serpentine locomotion with robotic snakes," IEEE Contr. Syst. Mag., vol. 22, no. 1, pp. 64-81, February 2002.

[6] G. P. Hicks, "Modeling and control of a snake-like serial-link structure," Ph.D. dissertation, North Carolina State University, 2003.

[7] M. Nilsson, "Serpentine locomotion on surfaces with uniform friction," in Proc. IEEE/RSJ Int. Conf. Intelligent Robots and Systems, 2004, pp. 1751-1755.

[8] P. Liljebäck, Ø. Stavdahl, and K. Y. Pettersen, "Modular pneumatic snake robot: 3D modelling, implementation and control," in Proc. 16th IFAC World Congress, July 2005.

[9] A. A. Transeth, R. I. Leine, Ch.. Glocker, and K. Y. Pettersen, "Non-smooth 3D modeling of a snake robot with frictional unilateral constraints," in Proc. IEEE Int. Conf. Robotics and Biomimetics, Kunming, China, Dec 2006, pp. 1181-1188.

[10] P. Liljebäck, K. Y. Pettersen, and Ø. Stavdahl, "Modelling and control of obstacle-aided snake robot locomotion based on jam resolution," in Proc. IEEE Int. Conf. Robotics and Automation, 2009, pp. 3807-3814.

[11] T. S. Parker and L. O. Chua, Practical Numerical Algorithms for Chaotic Systems. Springer-Verlag, 1989.

[12] E. R. Westervelt, J. W. Grizzle, C. Chevallereau, J. H. Choi, and B. Morris, Feedback Control of Dynamic Bipedal Robot Locomotion. CRC Press, 2007.

[13] P. Liljebäck, K. Y. Pettersen, Ø. Stavdahl, and J. T. Gravdahl, "Controllability analysis of planar snake robots influenced by viscous ground friction," in Proc. IEEE/RSJ Int. Conf. Intelligent Robots and Systems, 2009 , to appear. 